\title{
A bleeding gastric ulcer caused by anisakiasis
}

\section{Kenta Hamada ${ }^{a}$, Noriya Uedo ${ }^{a}$, Yasuhiko Tomita ${ }^{b}$, Hiroyasu lishi ${ }^{a}$}

Department of Gastrointestinal, Osaka Medical Center for Cancer and Cardiovascular Diseases, Osaka, Japan

A man in his 70 s presented to the emergency unit for hematemesis. His recent blood test showed normal liver and renal function, and serum anti-Helicobacter pylori (H. pylori) IgG antibody was negative. He took neither steroids nor nonsteroidal anti-inflammatory drugs (NSAIDs). Emergency endoscopy revealed a bleeding gastric ulcer in the greater curvature of the gastric body (Fig. 1A). Endoscopic hemostasis was performed using a combination of hemoclips and soft coagulation using hot biopsy forceps. Next day, second-look endoscopy revealed an Anisakis larva (Fig. 1B) on the edge of the ulcer. The larva was removed with biopsy forceps (Fig. 1C). He said that he ate pickled mackerel fish 5 days before hematemesis.

Anisakiasis is a human parasitic disease caused by eating undercooked or raw fish such as cod, salmon, herring or mackerel infected with third-stage Anisakis larvae [1,2]. Endoscopic appearance includes erythema, erosions, ulcerations, and submucosal tumor-like mass [2]. However, the incidence of bleeding gastric ulcers is low at $0.5 \%$ [3]. As Japanese dishes become popular around the world, consumption of raw fish food, sushi or sashimi, is increasing. We should be aware that anisakiasis can be a cause of non-H. pylori and non-NSAID peptic ulcer and bleeding.

Department of ${ }^{\mathrm{a}}$ Gastrointestinal Oncology (Kenta Hamada, Noriya Uedo, Hiroyasu Iishi) bathology (Yasuhiko Tomita), Osaka Medical Center for Cancer and Cardiovascular Diseases, Osaka, Japan

Conflict of Interest: None

Correspondence to: Noriya Uedo, Department of Gastrointestinal Oncology, Osaka Medical Center for Cancer and Cardiovascular Diseases, 1-3-3 Nakamichi Higashinari-ku, Osaka 537-8511, Japan, Tel.: +81 66972 1181, Fax: +81 66981 4067,

e-mail: uedou-no@mc.pref.osaka.jp

Received 05 April 2016; accepted 10 April 2016; published online 25 April 2016

DOI: http://dx.doi.org/10.20524/aog.2016.0039

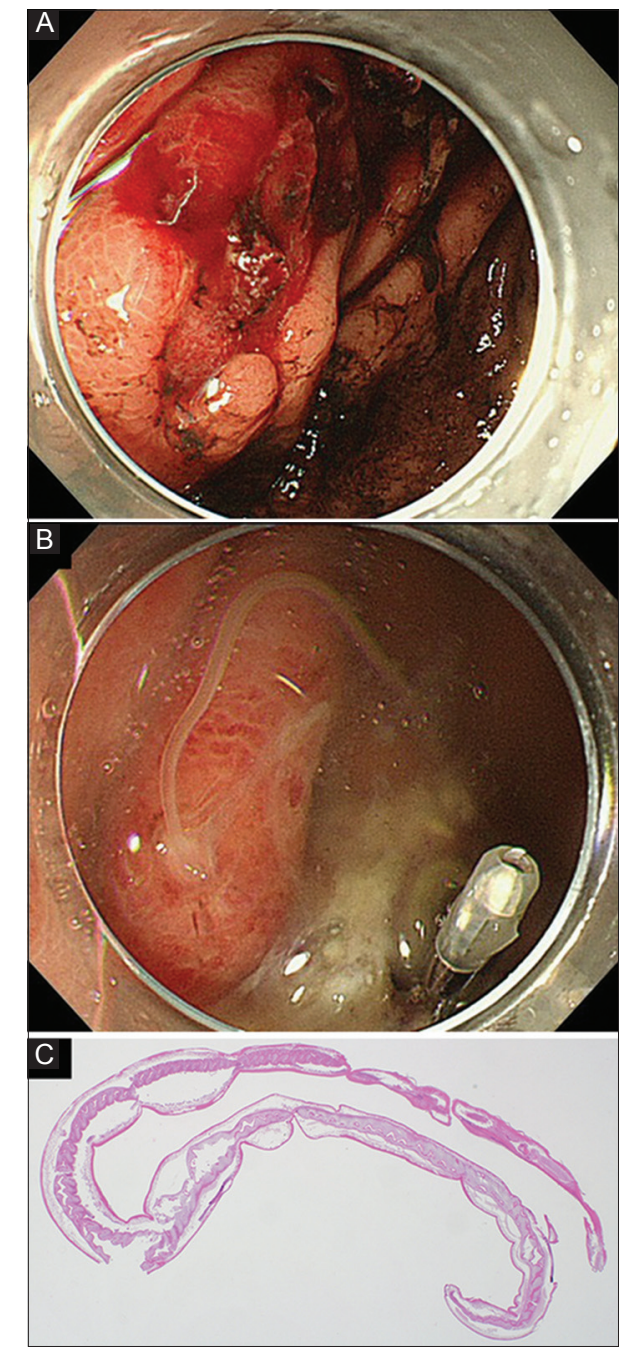

Figure 1 (A) Emergency endoscopy revealed a bleeding gastric ulcer in the greater curvature of the gastric body. (B) Second-look endoscopy revealed an Anisakis larva on the edge of the ulcer. (C) Histological examination showed the whole longitudinal body of an Anisakis larva

\section{References}

1. Oshima T. Anisakiasis - is the sushi bar guilty? Parasitol Today 1987;3:44-48.

2. Madi L, Ali M, Legace-Wiens P, Duerksen DR. Gastrointestinal manifestations and management of anisakiasis. Can J Gastroenterol 2013;27:126-127.

3. Goto Y, Takahashi N, Yoshimitsu M, Matano Y. A case of gastric anisakiasis with hemorrhagic gastric ulcer and ulcerative scarring [Article in Japanese]. Nihon Shokakibyo Gakkai Zasshi 2014;111:2021-2024. 